\title{
Innate chemical, but not visual, threat cues have been co-opted as unconditioned stimulus for social fear learning in zebrafish
}

\author{
Julia S. Pinho $^{1,2}$ | Marisa Castilho ${ }^{3}$ | Joao S. Sollari ${ }^{1,5}$ | Rui F. Oliveira ${ }^{1,2,4}$ (1)
}

\author{
${ }^{1}$ Integrative Behavioral Biology Laboratory, \\ Instituto Gulbenkian de Ciência, Oeiras, \\ Portugal \\ ${ }^{2}$ Department of Biosciences, ISPA-Instituto \\ Universitário, Lisboa, Portugal \\ ${ }^{3}$ Department of Physiology, School of \\ Biological Sciences, Universidade Federal do \\ Paraná (UFPR), Curitiba, Brazil \\ ${ }^{4}$ Champalimaud Research, Lisboa, Portugal \\ ${ }^{5}$ Instituto Nacional de Estatística, Lisboa, \\ Portugal
}

\section{Correspondence}

Rui F. Oliveira, Integrative Behavioral Biology Laboratory, Instituto Gulbenkian de Ciência, Oeiras, Portugal.

Email: ruiol@ispa.pt

\section{Funding information}

Fundação para a Ciência e a Tecnologia, Grant/Award Numbers: PTDC/BIAANM/0810/2014, SFRH/BD/97442/2013

\begin{abstract}
Animals can use social information to detect threat in the environment. In particular, social learning allows animals to learn about dangers without incurring in the costs of trial-and-error learning. In zebrafish, both chemical and visual social cues elicit an innate alarm response, which consists of erratic movement followed by freezing behavior. Injured zebrafish release an alarm substance from their skin that elicits the alarm response. Similarly, the sight of conspecifics displaying the alarm response can also elicit the expression of this response in observers. In this study, we investigated if these social cues of danger can also be used by zebrafish as unconditioned stimulus (US) in learning. We found that only the chemical cue was effective in the social fear conditioning. We suggest that this differential efficacy of social cues results from the fact that the alarm cue is a more reliable indicator of threat, than the sight of an alarmed conspecific. Therefore, although multiple social cues may elicit innate responses not all have been evolutionarily co-opted to act as US in associative learning. Furthermore, the use of the expression of the immediate early genes as markers of neuronal activity showed that chemical social fear conditioning is paralleled by a differential activation of the olfactory bulbs and by a different pattern of functional connectivity across brain regions involved in olfactory processing.
\end{abstract}

\section{KEYWORDS}

alarm substance, observational conditioning, olfactory system, social fear conditioning, social learning, zebrafish

\section{1 | INTRODUCTION}

A key component of Darwinian fitness is the ability of animals to detect and respond to the presence of danger in the environment, namely predators. Given that typically threat cues used by animals to detect danger have some overlap with background ambient noise in the sensory modality used to monitor the environment (eg., an individual may have to decide if a rustle in the grass indicates the presence of a predator or if it is just the wind), according to signal detection theory, individuals need to set a signal detection threshold that they use to make a decision that they are in the presence of a threat and activate the appropriate behavioral response. ${ }^{1,2}$ If individuals set a high threshold they will fail to detect a real threat frequently (miss), but they will activate few false alarms. On the other hand, if they set a low threshold they will miss fewer real threats, but at the cost of more frequent false alarms. Therefore, there is a trade-off between misses and false alarms and the setting of a threat detection threshold is critical for survival. ${ }^{3}$ Group living animals can use social information to detect a threat in the environment, and it has been demonstrated that the above-mentioned trade-off between misses and false alarms 
present in individual decision-makers can be overcome in a group of decision-makers using a quorum decision rule. ${ }^{4}$ Therefore, the use of social information in threat perception allows to overcome this basic trade-off in individual signal detection theory. Thus, the use of social information in the threat perception is expected to be widespread in social living organisms.

There is ample evidence that animals indeed use social information to modulate their threat perception. The exposure to social cues signaling threat, such as the sight, sound or smell of an alarmed conspecific usually triggers a fear response (eg., ${ }^{5-7}$ ), a phenomenon known as social contagion of fear. ${ }^{8,9}$ Conversely, the presence of a nonalarmed familiar conspecific may signal safety and it has been shown to attenuate fear response, which has been termed social buffering of fear (eg., ${ }^{10-12}$ ). Moreover, these social cues of threat can be used as unconditioned stimulus (US) in a classic conditioning paradigm, such that when paired with a conditioned stimulus (CS) may reinforce the establishment of conditioned fear responses to this CS, a phenomenon referred to as social fear learning (aka vicarious fear learning, vicarious aversive conditioning, or observational fear learning. ${ }^{13,14}$ One of the first documented cases of social fear learning was described in rhesus monkeys (Macaca mulatta) in which naïve individuals have no fear of snakes, but acquire a fear response toward snakes after observing a demonstrator reacting fearfully to a snake. ${ }^{15,16}$ Although this phenomenon was initially termed observational conditioning, it is not restricted to visual cues, and examples of social fear learning have been documented with odor cues, such as alarm pheromones in fish, ${ }^{17}$ or acoustic cues, such as mobbing calls in birds, ${ }^{18}$ being used as US. The ubiquity of social fear learning across different taxa and using different sensory modalities reflects its adaptive importance, since it allows individuals to learn about threat without using trial-and-error learning in an ecological domain, where the cost of misses would be very high, most probably death. ${ }^{19}$

Zebrafish uses both chemical and visual social threat cues to assess the presence of danger in the environment, and responds to these with an innate alarm response, which consists of erratic movement followed by freezing behavior. Like in many other fish species, injured zebrafish release an alarm substance from their skin into the water that is detected through olfaction eliciting an alarm response. ${ }^{20,21}$ The alarm substance, originally termed Schreckstoff by Karl von Frisch, and first described in minnows (Phoxinus phoxinus) ${ }^{22}$ is produced in specialized epidermal club cells, and is released upon skin injury. ${ }^{23}$ The molecular identity of the alarm substance has not been yet clearly established, but the available evidence suggests it is a mixture of compounds, and two putative active compounds have been suggested so far: hypoxanthine-3 $\mathrm{N}$-oxide and the glycosaminoglycan chondroitin. ${ }^{24-28}$ The sight of conspecifics displaying the alarm response can also elicit the expression of this response in observer zebrafish. ${ }^{29}$ Although there have been previous publications reporting socially learned alarm response in zebrafish, a closer examination of the original findings shows some weaknesses, namely the use of very small sample sizes, the use of group behavioral measures rather than individual ones, ignoring individual variation in the response and the use of a vertical index as the only behavioral measure of the alarm response ignoring key components of the response, such as the erratic movement and the freezing behavior. ${ }^{30,31}$ Despite these weaknesses in this seminal work, the occurrence of social transmission of fear in zebrafish has been propagated in secondary sources in the literature and given the attention that this field of research has been receiving recently it needs an urgent reassessment.

Here, we examined the efficacy of two social cues of danger (alarm substance and alarmed conspecifics) as US in social fear learning in zebrafish, and described the neuronal mechanisms involved, using expression of the immediate early genes as reporters of neuronal activity. Immediate-early genes (IEGs) are transiently expressed in neurons in response to neural activity, and hence they have been widely used as neural activity markers in studies of behavior and cognition (e.g. ${ }^{32,33}$ ). The two most commonly used IEGs to map neuronal activity, $c$-fos and egr1, encode transcription factors that regulate the expression of downstream target genes (aka late-response genes), which in turn regulate neuronal physiology. ${ }^{34-37}$ In particular, the expression of $c$-fos and egr1 have been related to learning and memory (eg., ${ }^{38-40}$ ). Bdnf and npas4 are two other IEG's whose expression has also been related to neural plasticity underlying long term memory formation (bdnf-synaptic plasticity ${ }^{41}$ npas4-homeostatic plasticity ${ }^{42}$ ). Thus, here we used the expression of these four different IEGs in order to capture possible different aspects of memory formation during social fear learning in zebrafish.

\section{2 | MATERIALS AND METHODS}

\subsection{Animals and housing}

Tubingen's adult male wild-type zebrafish (Danio rerio) $(n=72)$ were bred and held at Instituto Gulbenkian de Ciência (IGC, Oeiras, Portugal). Fish were kept in a recirculation system (ZebraTec, 93 Tecniplast) at $28^{\circ} \mathrm{C}$ with 14 light: 10 darkness photoperiod until 4 months of age. The water system was maintained at less than $0.2 \mathrm{ppm}$ nitrites, $50 \mathrm{ppm}$ nitrates and $0.01 \mathrm{ppm}$ ammonia, while $\mathrm{pH}$ and conductivity were maintained at 7 and $700 \mu \mathrm{Scm}^{-1}$, respectively. Fish were fed twice a day with commercial food flakes (Bionautic) and Artemia salina.

\section{2 | Experimental protocol}

In the innate response to threat experiment, each animal was exposed to one of four treatments for 5 minutes: alarm substance (alarm), pretrained conspecific (conspecific), distilled water (control) and light. The threat response is a stereotyped behavior where adult zebrafish exhibit erratic movement and freezing. Erratic movement is characterized by multiple darts (fast acceleration bouts and stochastic changes in direction) and is normally the first response to danger. ${ }^{43}$ Freezing is a complete cessation of movement (except for gills and eyes), while the fish is at the bottom of the tank and showing abundant opercular movements (respiration/gill movements). ${ }^{43}$ 
A behavioral paradigm was designed to characterize chemical and visual social fear learning in zebrafish. In visual social fear learning (Figure 1(A)), a demonstrator fish was trained to pair a light (CS) with alarm substance (US) (conditioned demonstrator, $\mathrm{CS}^{+} \mathrm{D}$ ) or distilled water (control; unconditioned demonstrator, $\mathrm{CS}^{-} \mathrm{D}$ ). This demonstrator training phase lasted for 3 days, with three trials/day. During this phase, the observer fish did not have contact with the demonstrator one. The observer training phase followed, during which the demonstrator and observer were presented with the CS for three trials/day for 3 days, which elicited a conditioned alarm response in the conditioned demonstrator, that should act as an US for the observer (conditioned observer, $\mathrm{CS}^{+} \mathrm{O}$ ). As a control treatment, a group of observers (unconditioned observers, $\mathrm{CS}^{-} \mathrm{O}$ ) were trained with unconditioned demonstrators (ie, that do not respond to the light [CS] with an alarm response). On the seventh day (trial test), the response of the observer toward the CS (light) was tested in the absence of the demonstrator. In chemical social fear learning (Figure 1(B)), the observer fish was subjected to water changes for 3 days in order to standardize conditions between experiments. During the training phase, the observer was trained (three trials/day for 3 days), in a similar way to the training of demonstrators in the previous experiment, to pair the light (CS) with the alarm substance (US) or with distilled water (control). On the trial test (7th day), the response of the observer toward the CS was tested in the absence of the alarm substance administration.

All fish were isolated in individual tanks 1-day before the experiments. Alarm substance (US) and distilled water (control) were introduced into the tanks using a plastic tube $(0.8 \mathrm{~mm}$ internal diameter, Kartell, UK) connected to a $5 \mathrm{~mL}$ syringe (Terumo, Japan). The alarm substance was prepared from skin extracts of zebrafish following a modified protocol described by ${ }^{20}$. The alarm substance donors were commercial wild-type zebrafish (half were males and half females) captured and quickly sacrificed by decapitation using surgical scissors. Light (CS) was presented at the side of the tank in the middle of the water column. The experiments were videotaped in side view. The behaviors were recorded using a multi-event recorder (Observer XT

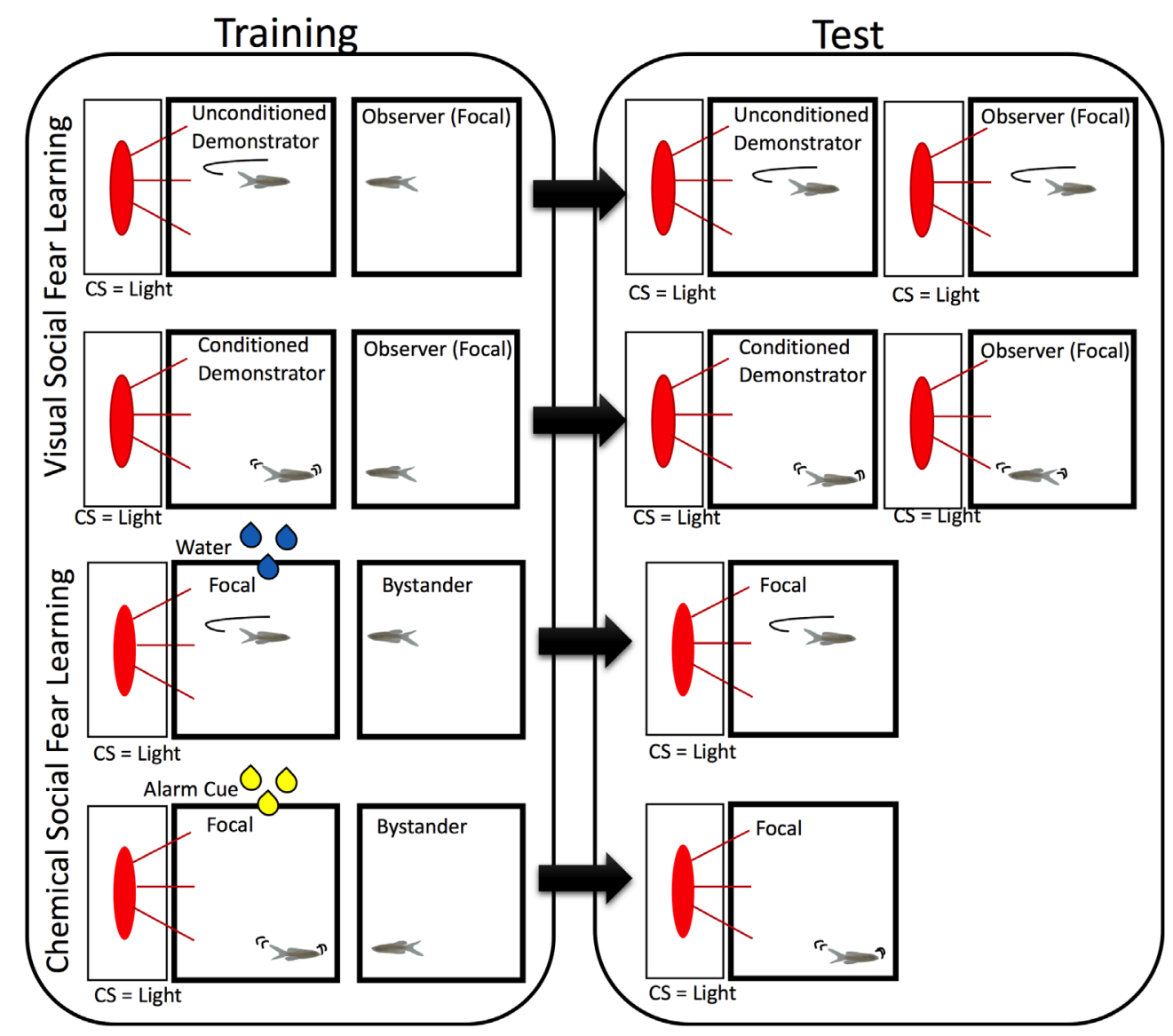

FIGURE 1 Experimental design of visual and chemical social fear learning. A, In visual social fear learning, the demonstrator was trained in the same way as the observer in the chemical social fear learning protocol (see below). During this period, an opaque partition was used to avoid visual access of the observer to the demonstrator. In the observer training phase, the light elicited an alarm response in the demonstrator, hence from the observer's perspective the light (CS) was paired with the sight of an alarmed conspecific (US). In the trial test, demonstrators were not present and the observer was exposed by themselves to the light (CS). B, In chemical social fear learning, after 3 days of water changes the training phase consisted of pairing a light (CS) with alarm substance (US) or distilled water (control). In the trial test, the CS alone was presented to evaluate if the fish have learned the association. The fish in the contiguous tank was used to standardize conditions between the visual and the chemical social fear learning experiments. CS, conditioned stimulus; US, unconditioned stimulus 
9, Noldus Technology) and an automatic tracking system (Ethovision XT 12, Noldus Technology).

\section{3 | Microdissection of the zebrafish brain}

After the behavioral experiment, the animals were quickly sacrificed with an excess of anesthesia (MS-222, Pharmaq; 300-400 ppm) followed by decapitation. The head was incorporated in Optical Cutting Temperature (OCT) (Tissue-Tek, Sakura, The Netherlands) and frozen at $-80^{\circ} \mathrm{C}$. Coronal head slices $(150 \mu \mathrm{m})$ were cut in a cryostat and stored at $-20^{\circ} \mathrm{C}$ on microscope slides (Thermo Scientific). Regions of interest were micropunched from the brain slices using a modified $27 \mathrm{G}$ needle (the bevel and the external diameter were removed), following a zebrafish brain atlas ${ }^{44}$ to localize their anatomical position. The following brain regions were micropunched: Olfactory bulb (OB), medial zone of dorsal telencephalic area (Dm: homolog of pallial amygdala in mammals), ${ }^{45-47}$ posterior zone of dorsal telencephalic area (Dp: homolog of olfactory cortex), ${ }^{48,49}$ ventral nucleus of ventral telencephalic area (Vv: homolog of septal formation in mammals ${ }^{48}$ and Habenula (Ha: homolog of mammalian lateral habenula). ${ }^{50}$ The samples were stored in Eppendorfs with $50 \mu \mathrm{L}$ of Qiazol (Quiagen) at $-80^{\circ} \mathrm{C}$.

\section{4 | Quantitative RNA expression of immediate early genes}

Total RNA from each brain microarea was extracted using RNeasy Lipid Tissue Mini Kit (Qiagen) and then stored at $-80^{\circ} \mathrm{C}$. The integrity of the RNA extracted was evaluated by Agilent 2100 Bioanalyzer (Agilent Technologies, UK). First-strand cDNA was prepared using iScript cDNA synthesis kit (Biorad) based on RNase $\mathrm{H}+$ and oligo (dT) and random hexamer primers and stored at $-20^{\circ} \mathrm{C}$. Quantitative PCR (qPCR) was performed in the ABI7900HT (Applied Biosystems, Life Technologies) using 384 well-plates, where cDNA, primers (Table S1) and SYBR green PCR master mix (Applied Biosystems, Life Technologies) were added. qPCR data were collected using Sequence Detection Systems (SDS 2.4) (Applied Biosystems, Life Technologies) with 1 cycle $95^{\circ} \mathrm{C}$ for 5 minutes; 40 cycles $95^{\circ} \mathrm{C}$ for 30 seconds, annealing temperature of the primers (Table S1) for 30 seconds and $72^{\circ} \mathrm{C}$ for 30 seconds). The threshold was defined by gene and a table of $\mathrm{Ct}$ values for each of the 384 reactions exported for data analysis. The $\mathrm{Ct}$ analysis was performed using $2^{\wedge}$ (Ct housekeeping-Ct target gene) assuming that the efficiency of the genes was $100 \%$. To validate this assumption, we measured the efficiency of each gene and we have only used primers with efficiencies above $90 \%$.

\subsection{Statistical analysis}

The behavioral effects of innate responses to alarm cues were analyzed using a nonparametric ANOVA (Kruskal-Wallis) followed by post-hoc tests, where equal variances were not assumed (Tamhane T2 post-hoc test). The occurrence of chemical social fear learning was tested using non-parametric $t$ tests (Mann-Whitney) for each measure (ie, erratic movement and freezing). The occurrence of visual social fear learning was tested using a nonparametric ANOVA (Kruskal-Wallis) followed by post-hoc comparisons, where equal variances were not assumed (Tamhane T2 post-hoc test) for each measure. The effects of social fear learning (trained animals with alarm cue or distilled water) and brain region ( $\mathrm{OB}, \mathrm{Dm}, \mathrm{Dp}, \mathrm{Vv}, \mathrm{Ha}$ ) in the expression of immediate early genes (c-fos, egr-1, bdnf, npas4) were tested using between-subject linear mixed models (LMMs) with the subject as a random effect. Parametric assumptions were checked using Shapiro-Wilk and Jarque-Bera adjusted multiplier tests (to test for normality), Bartlett, Levene and Fligner-Killeen tests (to test for homoscedasticity), and plots of the residuals, fitted values and estimated random effects in the LMM. Gene expression data were log-transformed before the analyses to fit parametric assumptions. Planned comparisons among social fear learning treatments within each brain nucleus and for each IEG were computed to test for differential activation of each brain region in response to social fear learning.

Functional connectivity among the sampled brain regions was tested with Pearson correlation matrices computed between the IEG expression for each pair of the brain region in social fear learning treatment. Two regions correlated positively indicate coactivation in response to that treatment, whereas two regions correlated negatively indicate reciprocal inhibition in response to the treatment. Quadratic assignment procedure (QAP) correlation tests with 5000 permutations were used to test for differences between the correlation matrices (that portrait coactivation/coinhibition among brain regions) for each treatment. ${ }^{51}$ In QAP tests a significant $P$-value is indicative of association between the matrices, that is, different patterns of functional connectivity captured by the matrices correspond to nonsignificant QAP test $P$-values.

The structure of the neural network composed of the sampled brain regions in this study was characterized using measures of centrality and cohesion. The centrality of each node in the network was measured using eigenvector centrality, which integrates every link a node receives with the relevance of each node of the network. The cohesion of the whole network was measured by density, which is the average of connections quantified for each network. Density was assessed using a bootstrap $t$ test approach with 5000 sub-samples.

Statistical analyses were performed on SPSS (version 22) and $R$ (version 3: www.R-project.org) using the following packages: car (Levene test), cluster (PAM), fBasics (Jarque - Bera test), Hmisc (correlations), lattice (heatmaps), multcomp (planned comparisons) and nlme (LMMs). The network analysis parameters were estimated using UCINET v. 6. Network representations were produced using Python.

\section{$3 \mid$ RESULTS}

\section{1 | Innate response to threat cues}

Both the alarm substance (alarm) and the sight of alarmed conspecifics (conspecific) induced a peak in swimming speed followed by a 
decrease, which corresponds to the erratic movement and freezing phases of the zebrafish alarm response (Figure 2(A,B)). A shorter distance from the bottom is also observed during the alarm response, and despite some inter-individual variation, the alarm response to both alarm cues is quite robust (Figure 2(A,B)). Importantly, neither distilled water (control) nor light triggered by themselves any such behavioral responses (Kruskall-Wallis test: erratic movement, $H=15.41, P<0.0001$; freezing, $H=55.17, P<0.0001$ ) and no differences between these two treatments were observed (multiple comparisons tests: erratic movement, $P=1$; freezing $P=1$ ). A single exposure to the alarm substance elicited an alarm response composed of $20 \%$ time in erratic movement and $80 \%$ in freezing (multiple comparisons tests: erratic movement, control vs alarm $P<0.0001$ and light vs. alarm $P<0.0001$; freezing: control vs alarm $P<0.0001$ and light vs alarm $P<0.0001$ ) (triangles, Figure $2(C)$ ). The sight of alarmed conspecific induced an alarm response (multiple comparisons tests: control vs conspecific, $P=0.005$ and light vs conspecific $P<0.005$ ) with a bimodal distribution in erratic movement (ie, with low and high responders) and freezing was almost absent (ie, there were no significant differences in the time in freezing between conspecific, light and control treatments; multiple comparisons tests: conspecific vs light $P=0.118$, conspecific vs control $P=0.005$ ) (Figure $2(C)$ ). Alarm and conspecific are innately different in the time spent in freezing (multiple comparisons test: $P<0.0001$ ). Control and light never elicited erratic movements or freezing (multiple comparisons tests: control vs light: erratic movement $P=1$, freezing $P=1$ ) (Figure $2(C)$ ).

\section{2 | Visual and chemical social fear learning}

In visual social fear learning, 50\% of the animals responded to the alarmed demonstrator on the first trial (US in this experiment) (black bars, Figure $3(A))$. Conditioned observers $\left(\mathrm{CS}^{+} \mathrm{O}\right)$ did not seem to have learned by observation that light (CS) predicts the alarmed conspecific (US), since there was no reduction in latency to respond to the CS along the training trials (black circles, Figure $3(B)$ ), and in the probe test they did not exhibit a conditioned alarm response towards the CS alone (multiple comparisons test: conditioned observer vs unconditioned observer, erratic movement $P=1$, freezing $P=1$; Figure $3(C)$, black circle vs white circle; conditioned observer vs conditioned demonstrator, erratic movement $P<0.0001$, freezing $P=0.11$; Figure $3(C)$, black circle vs black triangle). This lack of the conditioned response in the conditioned observers was not due to a failure of the conditioning protocol of demonstrators since conditioned demonstrators showed the erratic movement conditioned alarm response to the CS (light) (multiple comparisons test: conditioned demonstrator vs unconditioned demonstrator, erratic movement $P<0.0001$, freezing $P=0.11$; black triangle vs white triangle, Figure $3(C)$ ). Unconditioned demonstrators (ie, trained with distilled water) and unconditioned observers (ie, trained with unconditioned demonstrators) did not exhibit alarm responses toward the CS and expressed similar responses in the probe test (multiple comparisons test: unconditioned demonstrator vs unconditioned observer, erratic movement $P=1$, freezing $P=1$; white circle vs white triangle, Figure 3(C)).

In chemical social fear learning, $100 \%$ of the animals exhibited the alarm response from the first trial onward (in gray, Figure 3(A)), and during training, they started to express the alarm response before the US is present from the third trial onwards (in full gray squares, Figure 3(D)). Animals exposed to distilled water (control group) did not express the alarm response all over the training phase (in empty gray squares, Figure 3(D)). In the probe test animals expressed the alarm response toward the CS alone, but not toward the control (erratic movement: $M W=11, P=0.0001$, freezing: $\mathrm{MW}=11, P<0.0001$ ).

These results show that although zebrafish has an innate response both to chemical and to visual social cues of danger, it only learns from chemical but not from visual cues. Since only the chemical social cue acted as a US, only the brains of animals from this
(A)

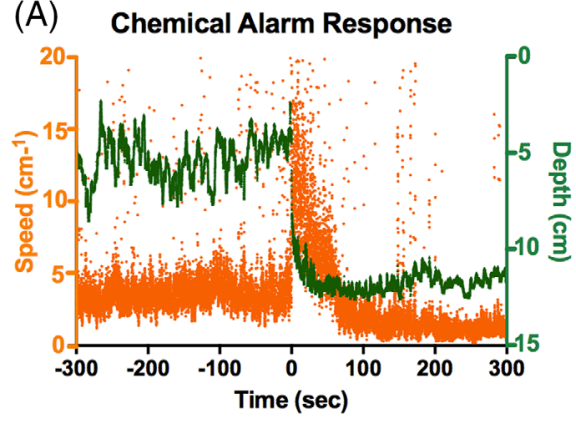

(B)

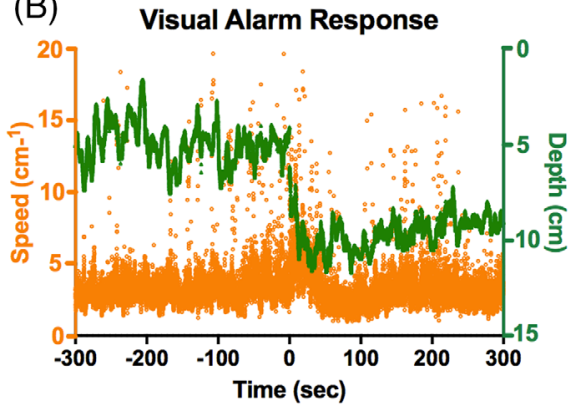

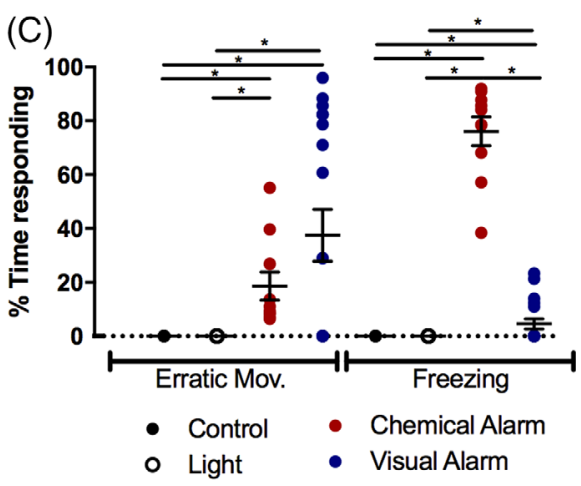

FIGURE 2 Innate response to visual (sight of alarmed conspecifics termed as conspecific) and chemical (alarm substance termed as alarm) social cues of threat, distilled water (control) and light. Speed (red) and depth (green) are plotted along time in response to alarm substance, A, and sight of alarmed conspecific, $\mathrm{B} ; \mathrm{t}=\mathrm{O}$ is when the social cue is delivered. The percentage of time in erratic movement and freezing was measured in all experimental treatments, $C$. * Represents $P$-value $<0.05$ 
(A)

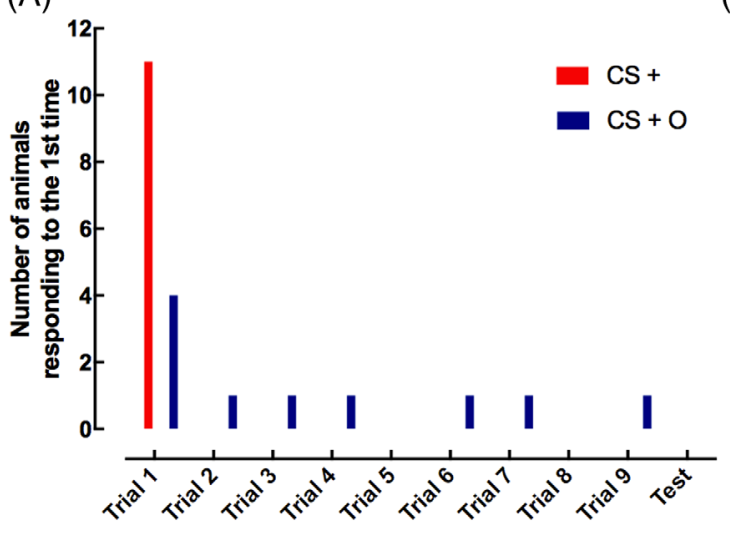

(C)

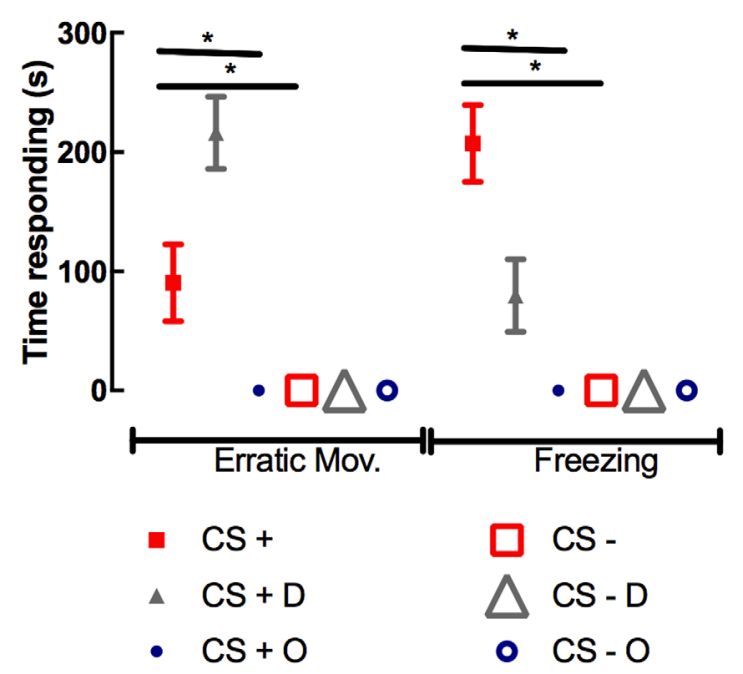

(B)

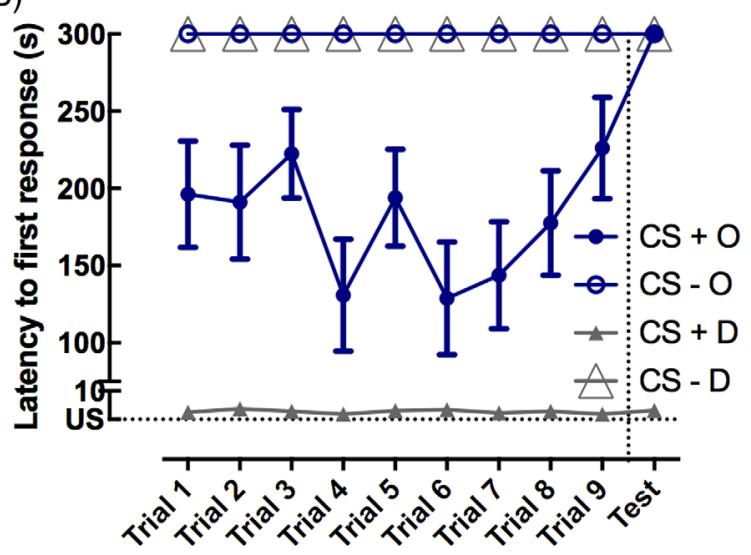

(D)

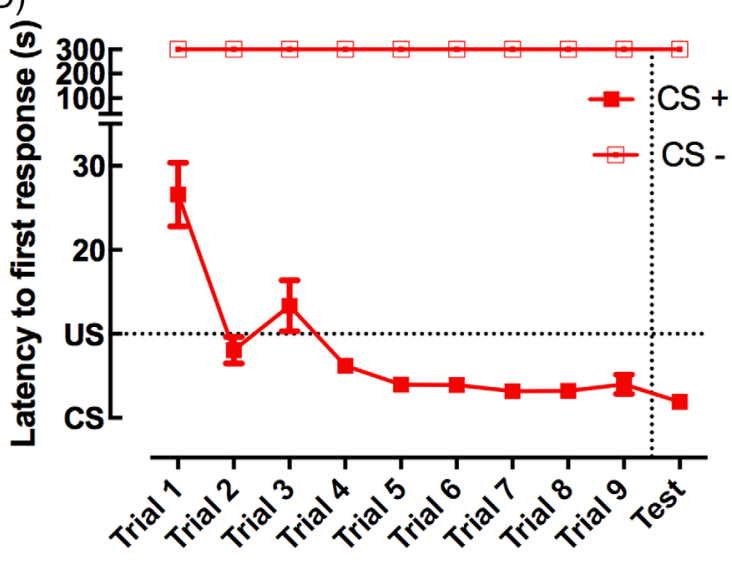

FIGURE 3 Chemical and visual social fear learning in zebrafish. A, Number of animals responding for the first time to CS in chemical social fear learning (gray bars) and visual social fear learning (black bars). B, Latency of the first response of conditioned ( $\mathrm{CS}^{+} \mathrm{O}$, black circle) and unconditioned ( $\mathrm{CS}^{-} \mathrm{O}$, white circle) observers towards the CS in the visual social fear learning paradigm (for comparison the latency of conditioned ( $\left(\mathrm{SS}^{+} \mathrm{D}\right.$, black triangles) and unconditioned [ $\mathrm{CS}^{-} \mathrm{D}$, white triangles] demonstrators towards $\mathrm{CS}$ are also shown). $\mathrm{C}$, Time responding with erratic movement (in sec) (left) and with freezing (right) during the trial test, by the same experimental groups described in B and D. D, Latency to the first response to $\mathrm{CS}\left(\mathrm{CS}^{+}\right.$, gray square) and control $\left(\mathrm{CS}^{-}\right.$, gray open square) in chemical social fear learning. Values are means $\pm \mathrm{SE}$. ${ }^{*}$ Represents $P$ value $<0.05$. CS, conditioned stimulus

experiment were collected to characterize the putative neural circuits underlying social fear learning.

\subsection{Neural correlates of social fear learning}

Transcriptional expression of immediate early genes (c-fos, egr-1, bdnf and npas4) was measured in brain regions of interest (OB, Dm, Dp, $\mathrm{V} v$ and $\mathrm{Ha}$ ) as markers of neuronal activation. Egr-1 and npas4 RNA expression levels (normalized to the housekeeping gene elf1a) in OBs in response to the CS+ in the probe test were significantly reduced when compared with their response to the CS- (egr1 ( $z=2.48 \pm 0.51, P=0.013$ ) and npas4 (2.19 $\pm 0.51, P=0.028$ ) (Figure 4). No other significant differences in RNA expression of IEGs were found in the sampled brain regions (Table S2).

\subsection{Changes in functional connectivity of brain regions of interest in response to social fear learning}

In chemical social fear conditioning, the coactivation matrices for CS treatment were significantly different from control for $c$-fos ( $r=0.51 P=0.186)$, egr-1 $(r=0.32 P=0.337)$, bdnf $(r=0.67 P=0.173)$ and npas4 $(r=0.23 P=0.385)$ (Figure 5$)$. The structure of the gene expression networks was characterized through density and cohesion. The density of gene expression induced by the CS was significantly lower than that induced by CS control for bdnf ( $t=1.77 P=0.041)$ and npas 4 ( $t=1.62 P=0.037$ ). The centrality of the different brain regions was also different between $\mathrm{CS}$ and control treatments for the different IEG's. 


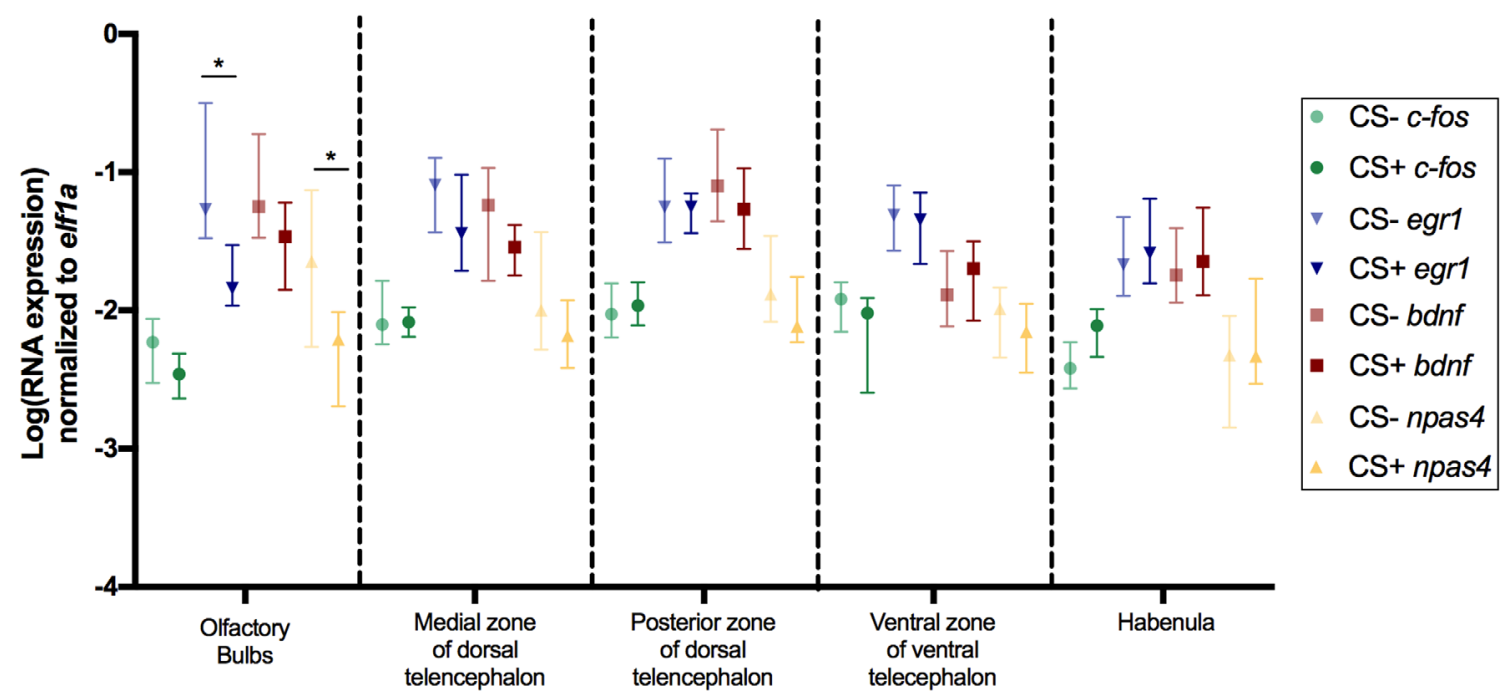

FIGURE 4 Transcriptional pattern of RNA expression of the $c$-fos (green), egr-1 (blue), bdnf (yellow) and npas4 (red) genes was measured after the probe test is response to CS (in dark colors) and to control (in light colors) across the brain regions of interest (OB, Dm, Dp, Vv and Ha). Values are median \pm interquartile ranges. ${ }^{*}$ Represents a significant difference between the indicated groups. CS, conditioned stimulus
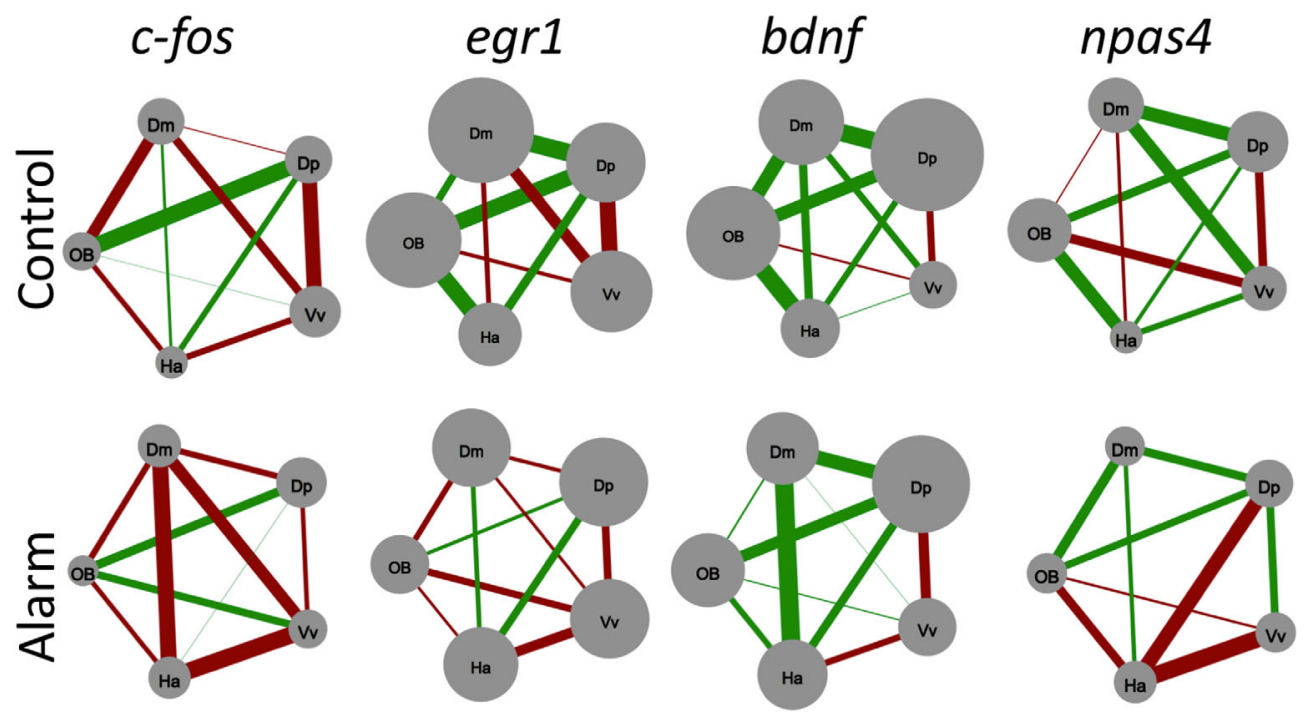

FIGURE 5 Functional connectivity across the brain regions of interest induced by chemical social fear learning (response to control in first row; response to CS in second row) inferred from the expression of different immediate early genes used as markers of neuronal activity (c-fos, first column; egr-1, second column; bdnf, third column; npas4, fourth column). The diameter of the circles delimiting each node indicates RNA expression level of each gene on node. Thickness of edges indicates the magnitude of the correlation coefficient ( $r$ ) between pairs of nodes and edges color represents the sign $($ positive $=$ green; negative $=$ red) of the correlation coefficient. CS, conditioned stimulus

\section{4 | DISCUSSION}

Our results demonstrate for the first time, that zebrafish learn a conditioned fear response using alarm substance but not the sight of an alarmed conspecific as a US. Thus, although zebrafish innately respond both to chemical and visual alarm cues, only chemical cues are efficient as an US in fear conditioning. These results suggest that chemical alarm cues have a higher threat value than the visual ones. Indeed, since the content of club cells (that produce the alarm substance) cannot be released voluntarily, and these cells are among the first to be damaged upon a predator attack (due to their superficial location in the epidermis), the release of the alarm substance is a reliable social cue for the presence of an active predator in the environment. $^{23,52}$ In contrast, behavioral alarm responses in conspecifics do not request a predator attack to have happened and must be more variable across individuals and hence less reliable. However, this result is somewhat surprising since social fear learning is highly adaptive, given the cost to learn by trial-and-error the consequences of interacting with threat sources (eg., predators). For instance, in the damselfish Acanthochromis polyacanthus, individuals that socially 
learned the odor of the predator increased their probability of surviving 5 -fold. ${ }^{53}$

Different sensory modalities can be used to detect alarm cues in the environment. Both chemical and visual alarm cues are well documented in fish. ${ }^{54,55}$ In 2001, a study showed that chemical cues seem to have a role in warning against the presence of a predator and visual cues in assessing the magnitude of risk in sculpin. ${ }^{56}$ While studies have shown that visual information is enough to elicit learning, ${ }^{57}$ others highlighted that in the absence of light, social recognition can still occur in damselfish ${ }^{58}$ indicating the importance of chemical cues to learning processes. The salience and the valence of the stimuli are important factors in learning success. Individuals learn faster with aversive cues, since the cost of receiving a punishment is higher than losing a reward. ${ }^{59}$ Also, the salience of a stimulus plays a role in learning abilities; high and low intensity shock, for instances, have different costs, ${ }^{60}$ and in zebrafish, the intensity of the alarm reaction varies directly with the concentration of the alarm substance that the fish is exposed to ${ }^{20}$. Moreover, it has been established that animals are evolutionary predisposed to learn some associations better than others, a phenomenon named prepared learning. ${ }^{61}$ Together these facts sug gest that chemical alarm cues are more reliable than visual ones, and hence became more salient and as a result, zebrafish became predisposed to learn better a fear-conditioned response triggered by a chemical US than by a visual US.

The neural mechanisms associated with chemical social fear learning were assessed through the characterization of the expression of a set of IEG's on regions of interest in the brain known to be involved in the processing of olfactory stimuli $\left(\mathrm{OB}, \mathrm{Dp}, \mathrm{Hb}\right.$ and $\mathrm{Vv}^{62}$ or in fear conditioning $\left(\mathrm{Dm}^{63}\right)$. Surprisingly, we only observed a differential neuronal response to social fear learning at the level of the OBs, and not at higher order processing areas of the olfactory circuit in zebrafish. In particular, we did not observe differential activation of the Dp, an area considered to be the teleost homolog of the mammalian olfactory cortex, ${ }^{48,49}$ and that has recently been shown to represent valence of odors in olfactory associative learning in zebrafish. ${ }^{64}$ Here it is important to note that while in Frank et $a^{64}$ calcium imaging was used allowing cellular resolution of the patterns of neuronal activation, in the present study we used qPCR expression of IEG's which are limited to a region of interest resolution. Thus, the most parsimonious explanation for the mismatch between the results presented here, and those of Frank et $a^{64}$ is that the changes in odor representation in neuronal subpopulations within Dp are not accompanied by an overall change in activity in Dp that can be captured by the level of expression of IEG's for the whole region. Moreover, this mismatch should be seen as an example of the limitation of the approach used here when interpreting negative results.

Despite of this mismatch, we have identified a decrease of activity in the OBs associated with social fear learning, suggesting the influence of a local disinhibitory mechanism. The OBs are a brain sensory area that is involved in the detection of olfactory cues in the environment, receiving projections from olfactory sensory neurons of the olfactory epithelium in a topographical fashion, and projecting, through mitral cells, which are second-order sensory neurons, to olfactory higher processing areas, such as the olfactory cortex in mammals. Interestingly, a large population of GABAergic interneurons (aka granule cells) that regulate the activity of mitral cells, is also present in the inner layer of the OBs in a ratio of 10:1 to mitral cells. ${ }^{62}$ Thus, our results most probably reflects a learninginduced reduction in the activity of these inhibitory granule cells. Given that, in zebrafish the OBs have also been described to process the innate response to alarm substance. ${ }^{65}$ This putative change in the activity of granule cells is most probably changing the odor computations already at the level of the OBs in an experiencedependent manner.

In fact, the involvement of the OBs in odor fear conditioning has also been reported in rodents, ${ }^{19}$ where they have also been implicated in appetitive odor learning. ${ }^{66}$ Moreover, antagonists of norepinephrine receptor in the OBs impair conditioned odor preference learning, memory recognition and odor identification, ${ }^{67-70}$ and OB circuits have also been shown to display functional plasticity, including long-term synaptic potentiation, ${ }^{71}$ adult neurogenesis ${ }^{72}$ and reconfiguration by neuromodulators. ${ }^{73}$ Together, these data support a role for the OBs as plastic brain nuclei involved in olfactory learning in zebrafish besides their sensory role. $^{74}$

Different patterns of functional connectivity among the studied brain regions of interest were also observed between chemically conditioned animals and their controls for all IEGs tested (c-fos, egr-1, bdnf, and npas4). The central areas in each network were also different between chemical social fear learning and its controls for all genes. In the Bdnf and npas4 networks, there was a significant decrease in the average number of connections in chemical-conditioned individuals in relation to their controls. These results are in agreement with the previous literature that has indicated a role for both bdnf and npas4 in learning and memory. Bdnf is a known neuromodulator in mammalian hippocampus acting on long-term potentiation (reviews ${ }^{75,76}$ ), whereas npas 4 has been implied in the formation of contextual memories in the hippocampus in rodents. ${ }^{77}$

Thus, chemical social fear learning in zebrafish seems to rely on a dishinibition of the OBs accompanied by changes in functional connectivity of the neural circuit processing odor information.

In summary, we have confirmed the occurrence of social fear learning in zebrafish only for chemical cues, and we have shown that it is paralleled by a differential activation of the OBs and by a different pattern of functional connectivity across brain regions involved in olfactory processing.

\section{ACKNOWLEDGMENTS}

The authors would like to thank Peter McGregor for detailed comments on a first draft of this manuscript, which was written during the January 2019 ISPA Advanced Course on Scientific Writing. This research was funded by the grant PTDC/BIA-ANM/0810/2014 from Fundação para a Ciência e a Tecnologia (FCT) awarded to Rui F. Oliveira Julia S. Pinho was supported by a FCT Ph.D. fellowship (SFRH/BD/97442/2013).Additional supporting information may be found online in the supporting information section at the end of this article. 


\section{CONFLICT OF INTEREST}

The authors declare that they have no conflict of interest

\section{AUTHOR CONTRIBUTIONS}

Rui F. Oliveira, Julia S. Pinho and Marisa Castilho designed the research; Julia S. Pinho performed the research; Julia S. Pinho carried out the molecular lab work; Julia S. Pinho and J. S. L. carried out the statistical analyses; Julia S. Pinho and Rui F. Oliveira wrote the paper and all authors critically revised the manuscript.

\section{ETHICS STATEMENT}

All experiments were performed in accordance with the relevant guidelines and regulations for the care and use of animals in research and approved by the competent Portuguese authority (Direção Geral de Alimentação e Veterinária, permit 008955).

\section{DATA AVAILABILITY STATEMENT}

The data that support the findings of this study are available from the corresponding author upon reasonable request.

\section{ORCID}

Rui F. Oliveira (D) https://orcid.org/0000-0003-1528-618X

\section{REFERENCES}

1. Wiley RH. Signal detection and animal communication. Adv Study Behav. 2006;36:217-247.

2. Wiley RH. In: Brumm $\mathrm{H}$, ed. Animal communication and Noise. New York: Springer-Verlag; 2013:7-30.

3. Oliveira RF, Faustino Al. Social information use in threat perception: social buffering, contagion and facilitation of alarm responses. Commun Integr Biol. 2017;10:1-5. https://doi.org/10.1080/ 19420889.2017.1325049.

4. Wolf M, Kurvers RHJM, Ward AJW, Krause S, Krause J. Accurate decisions in an uncertain world: collective cognition increases true positives while decreasing false positives. Proc $R$ Soc B Biol Sci. 2013; 280:1-9. https://doi.org/10.1098/rspb.2012.2777.

5. Kim EJ, Kim ES, Covey E, Kim JJ. Social transmission of fear in rats: the role of $22-\mathrm{kHz}$ ultrasonic distress vocalization. PLoS One. 2010;5: 1-8. https://doi.org/10.1371/journal.pone.0015077.

6. Pereira AG, Cruz A, Lima SQ, Moita MA. Silence resulting from the cessation of movement signals danger. Curr Biol. 2012;22:R627R628. https://doi.org/10.1016/j.cub.2012.06.015.

7. Inagaki $\mathrm{H}$, Kiyokawa $\mathrm{Y}$, Tamogami $\mathrm{S}$, Watanabe $\mathrm{H}$, Takeuchi $\mathrm{Y}$, Mori Y. Identification of a pheromone that increases anxiety in rats. Proc Natl Acad Sci. 2014;111:18751-18756. https://doi.org/10.1073/ pnas.1414710112.

8. Dezecache G, Jacob P, Grèzes J. Emotional contagion: its scope and limits. Trends Cogn Sci. 2015;19:297-299. https://doi.org/10.1016/j. tics.2015.03.011.

9. Keum S, Shin H-S. Rodent models for studying empathy. Neurobiol Learn Mem. 2016;135:22-26. https://doi.org/10.1016/j.nlm.2016. 07.022 .

10. Smith AS, Wang Z. Hypothalamic oxytocin mediates social buffering of the stress response. Biol Psychiatry. 2014;76:281-288. https://doi. org/10.1016/j.biopsych.2013.09.017.

11. Edgar J, Held S, Paul E, Pettersson I, I'Anson Price R, Nicol C. Social buffering in a bird. Anim Behav. 2015;105:11-19. https://doi.org/10. 1016/j.anbehav.2015.04.007.
12. Kiyokawa Y, Honda A, Takeuchi Y, Mori Y. A familiar conspecific is more effective than an unfamiliar conspecific for social buffering of conditioned fear responses in male rats. Behav Brain Res. 2014;267: 189-193. https://doi.org/10.1016/j.bbr.2014.03.043.

13. Olsson A, Phelps EA. Social learning of fear. Nat Neurosci. 2007;10: 1095-1102. https://doi.org/10.1038/nn1968.

14. Debiec J, Olsson A. Social fear learning: from animal models to human function. Trends Cogn Sci. 2017;21:546-555. https://doi.org/10. 1016/j.tics.2017.04.010.

15. Mineka S, Davidson M, Cook M, Keir R. Observational conditioning of snake fear in rhesus monkeys. J Abnorm Psychol. 1984;93:355-372. https://doi.org/10.1037/0021-843X.93.4.355.

16. Cook M, Mineka S. Observational conditioning of fear to fearrelevant versus fear-irrelevant stimuli in rhesus monkeys. J Abnorm Psychol. 1989;98:448-459. https://doi.org/10.1037/0021-843X.98. 4.448.

17. Brown GE, Chivers DP. In: Brown C, Laland K, Krause J, eds. Fish Cognition and Behavior. Oxford: Blackwell Publ; 2006:49-69.

18. Curio E. In: Zentall TR, Galef BG Jr, eds. Social learning: Psychological and Biological Perspectives. Mahwah, NJ: Lawrence Erlbaum Associates, Inc; 1988:75-97.

19. Jones SV, Stanek-Rattiner L, Davis M, Ressler KJ. Differential regional expression of brain-derived neurotrophic factor following olfactory fear learning. Learn Mem. 2007;14:816-820. https://doi.org/10. 1101/Im.781507.

20. Speedie N, Gerlai R. Alarm substance induced behavioral responses in zebrafish (Danio rerio). Behav Brain Res. 2008;188:168-177. https:// doi.org/10.1016/j.bbr.2007.10.031.

21. Jesuthasan SJ, Mathuru AS. The alarm response in zebrafish: innate fear in a vertebrate genetic model. J Neurogenet. 2008;22:211-228. https://doi.org/10.1080/01677060802298475.

22. Frisch VKV. Über einen Schreckstoff der Fischhaut und seine biologische Bedeutung. Z Vergl Physiol. 1942;29:46-145. https://doi.org/10. 1007/BF00304445.

23. Smith RJF. Alarm signals in fishes. Rev Fish Biol Fish. 1992;2:33-63. https://doi.org/10.1007/BF00042916.

24. Lebedeva N, Malyukina G, Kasumyan A. The natural repellent in the skin of cyprinids. J Ichthyol. 1975;15:472-480.

25. Pfeiffer W, Riegelbauer G, Meier G, Scheibler B. Effect of hypoxanthine-3(N)-oxide and hypoxanthine- $1(\mathrm{~N})$-oxide on central nervous excitation of the black tetra Gymnocorymbus ternetzi (Characidae, Ostariophysi, Pisces) indicated by dorsal light response. J Chem Ecol. 1985;11:507-523. https://doi.org/10.1007/BF00989562.

26. Brown GE, Adrian JC, Smyth E, et al. Ostariophysan alarm pheromones: laboratory and field tests of the functional significance of nitrogen oxides. J Chem Ecol. 2000;26:139-154. https://doi.org/10. 1023/A:1005445629144.

27. Mathuru AS, Kibat C, Cheong WF, et al. Chondroitin fragments are odorants that trigger fear behavior in fish. Curr Biol. 2012;22:538544. https://doi.org/10.1016/j.cub.2012.01.061.

28. Parra KV, Adrian JC Jr, Gerlai R. The synthetic substance hypoxanthine 3-N-oxide elicits alarm reactions in zebrafish (Danio rerio). Behav Brain Res. 2009;205:336-341. https://doi.org/10.1016/j.bbr.2009. 06.037.

29. Fernandes Silva P, Garcia de Leaniz C, Luchiari AC. Fear contagion in zebrafish: a behaviour affected by familiarity. Anim Behav. 2019;153: 95-103. https://doi.org/10.1016/j.anbehav.2019.05.004.

30. Hall D, Suboski MD. Visual and olfactory stimuli in learned release of alarm reactions by zebra danio fish (Brachydanio rerio). Neurobiol Learn Mem. 1995;63:229-240. https://doi.org/10.1006/nlme.1995.1027.

31. Suboski MD, Bain S, Carty AE, McQuoid LM, et al. Alarm reaction in acquisition and social transmission of simulated-predator recognition by zebra danio fish (Brachydanio rerio). J Comp Psychol. 1990;104: 101-112. https://doi.org/10.1037/0735-7036.104.1.101. 
32. Mello CV, Vicario DS, Clayton DF. Song presentation induces gene expression in the songbird forebrain. Proc Natl Acad Sci. 1992;89: 6818-6822. https://doi.org/10.1073/pnas.89.15.6818.

33. Mello CV, Ribeiro S. ZENK protein regulation by song in the brain of songbirds. J Comp Neurol. 1998;393:426-438. https://doi.org/10. 1002/(SICI)1096-9861(19980420)393:4<426::AID-CNE3>3.0.CO;2-2.

34. Curran T, Morgan Jl. Memories of fos. Bioessays. 1987;7:255-258. https://doi.org/10.1002/bies.950070606.

35. O'Donovan KJ, Tourtellotte WG, Milbrandt J, Baraban JM. The EGR family of transcription-regulatory factors: Progress at the interface of molecular and systems neuroscience. Trends Neurosci. 1999;22:167173. https://doi.org/10.1016/S0166-2236(98)01343-5.

36. Tischmeyer W, Grimm R. Activation of immediate early genes and memory formation. Cell Mol Life Sci. 1999;55:564-574. https://doi. org/10.1007/s000180050315.

37. Pinaud R. Experience-dependent immediate early gene expression in the adult central nervous system: evidence from enrichedenvironment studies. Int J Neurosci. 2004;114:321-333. https://doi. org/10.1080/00207450490264142.

38. Rosen JB, Fanselow MS, Young SL, Sitcoske M, Maren S. Immediateearly gene expression in the amygdala following footshock stress and contextual fear conditioning. Brain Res. 1998;796:132-142. https:// doi.org/10.1016/S0006-8993(98)00294-7.

39. Guzowski JF, Setlow B, Wagner EK, McGaugh JL. Experiencedependent gene expression in the rat hippocampus after spatial learning: a comparison of the immediate-early genes arc, c- fos, and zif268. J Neurosci. 2001;21:5089-5098. https://doi.org/10.1523/ jneurosci.21-14-05089.2001.

40. Minatohara K, Akiyoshi M, Okuno $\mathrm{H}$. Role of immediate-early genes in synaptic plasticity and neuronal ensembles underlying the memory trace. Front Mol Neurosci. 2016;8:1-11. https://doi.org/10.3389/ fnmol.2015.00078.

41. Leal G, Comprido D, Duarte CB. BDNF-induced local protein synthesis and synaptic plasticity. Neuropharmacology. 2014;76:639-656. https://doi.org/10.1016/j.neuropharm.2013.04.005.

42. Lin Y, Bloodgood BL, Hauser JL, et al. Activity-dependent regulation of inhibitory synapse development by Npas4. Nature. 2008;455: 1198-1204. https://doi.org/10.1038/nature07319.

43. Kalueff AV, Gebhardt M, Stewart AM, et al. Towards a comprehensive catalog of zebrafish behavior 1.0 and beyond. Zebrafish. 2013;10: 70-86. https://doi.org/10.1089/zeb.2012.0861.

44. Wullimann MF, Rupp B, Reichert H. Neuroanatomy of the Zebrafish Brain. Basel: Birkhäuser Basel; 1996.

45. Portavella M, Torres B, Salas C. Avoidance response in goldfish: emotional and temporal involvement of medial and lateral telencephalic pallium. J Neurosci. 2004;24:2335-2342.

46. Northcutt RG. Connections of the lateral and medial divisions of the goldfish telencephalic pallium. J Comp Neurol. 2006;494:903-943.

47. Martín I, Gomez A, Salas C, Puerto A, Rodriguez F. Dorso-medial pallium lesions impair taste aversion learning in goldfish. Neurobiol Learn Mem. 2011;96:297-305.

48. Wullimann MF, Mueller T. Teleostean and mammalian forebrains contrasted: evidence from genes to behavior. J Comp Neurol. 2004;475: 143-162.

49. Mueller T, Dong Z, Berberoglu MA, Guo S. The dorsal pallium in zebrafish, Danio rerio (Cyprinidae, Teleostei). Brain Res. 2011;1381: 95-105.

50. Amo R, Aizawa H, Takahoko M, et al. Identification of the zebrafish ventral habenula as a homolog of the mammalian lateral habenula. J Neurosci. 2010;30:1566-1574. https://doi.org/10.1523/jneurosci. 3690-09.2010.

51. Borgatti SP, Everett MG, Johnson JC. Analyzing Social Networks. Global J Hum-Soc Sci. 2016;16:1-2.

52. Chivers DP, Wisenden BD, Hindman CJ, et al. Epidermal 'alarm substance' cells of fishes maintained by non-alarm functions: possible defence against pathogens, parasites and UVB radiation. Proc Biol Sci B. 2007;274:2611-2619. https://doi.org/10.1098/rspb.2007.0709.

53. Manassa RP, McCormick MI. Social learning and acquired recognition of a predator by a marine fish. Anim Cogn. 2012;15:559-565. https:// doi.org/10.1007/s10071-012-0484-z.

54. Brown GE, Magnavacca G. Predator inspection behaviour in a characin fish: an interaction between chemical and visual information? Ethology. 2003;109:739-750. https://doi.org/10.1046/j.1439-0310. 2003.00919.x.

55. Elvidge CK, Brown GE. Visual and chemical prey cues as complementary predator attractants in a tropical stream fish assemblage. Int J Zool. 2012;2012:1-7. https://doi.org/10.1155/2012/510920.

56. Chivers DP, Mirza RS, Bryer PJ, Kiesecker JM. Threat-sensitive predator avoidance by slimy sculpins: understanding the importance of visual versus chemical information. Can J Zool. 2001;79:867-873. https://doi.org/10.1139/z01-049.

57. Ferrari MCO, Trowell JJ, Brown GE, Chivers DP. The role of learning in the development of threat-sensitive predator avoidance by fathead minnows. Anim Behav. 2005;70:777-784. https://doi.org/10.1016/j. anbehav.2005.01.009.

58. Manassa RP, McCormick MI, Chivers DP, Ferrari MCO. Social learning of predators in the dark: understanding the role of visual, chemical and mechanical information. Proc R Soc B Biol Sci. 2013;280:1-8. https://doi.org/10.1098/rspb.2013.0720.

59. Steel A, Silson EH, Stagg CJ, Baker $\mathrm{Cl}$. The impact of reward and punishment on skill learning depends on task demands. Sci Rep. 2016;6:19. https://doi.org/10.1038/srep36056.

60. Rumbaugh DM, King JE, Beran MJ, Washburn DA, Gould KL. A salience theory of learning and behavior: with perspectives on neurobiology and cognition. Int J Primatol. 2007;28:973-996. https://doi. org/10.1007/s10764-007-9179-8.

61. Dunlap AS, Stephens DW. Experimental evolution of prepared learning. Complex Int. 2000;8:11750-11755. https://doi.org/10.1073/ pnas.1404176111.

62. Kermen F, Franco LM, Wyatt C, Yaksi E. Neural circuits mediating olfactory-driven behavior in fish. Front Neural Circuits. 2013;7:1-9. https://doi.org/10.3389/fncir.2013.00062.

63. Lal $\mathrm{P}$, Tanabe $\mathrm{H}$, Suster $\mathrm{ML}$, et al. Identification of a neuronal population in the telencephalon essential for fear conditioning in zebrafish. BMC Biol. 2018;16:1-18. https://doi.org/10.1186/s12915-0180502-y.

64. Frank T, Mönig NR, Satou C, Higashijima SI, Friedrich RW. Associative conditioning remaps odor representations and modifies inhibition in a higher olfactory brain area. Nat Neurosci. 2019;22:1844-1856. https://doi.org/10.1038/s41593-019-0495-z.

65. Enjin A, Suh GSB. Neural mechanisms of alarm pheromone signaling. Mol Cells. 2013;35:177-181. https://doi.org/10.1007/s10059-0130056-3.

66. Chen CF, Barnes DC, Wilson DA. Generalized vs. stimulus-specific learned fear differentially modifies stimulus encoding in primary sensory cortex of awake rats. J Neurophysiol. 2011;106:3136-3144. https://doi.org/10.1152/jn.00721.2011.

67. Guerin D, Peace ST, Didier A, et al. Noradrenergic neuromodulation in the olfactory bulb modulates odor habituation and spontaneous discrimination. Behav Neurosci. 2008;122:816-826. https://doi.org/ 10.1037/a0012522.

68. Escanilla O, Alperin S, Youssef M, Ennis M, Linster C. Noradrenergic but not cholinergic modulation of olfactory bulb during processing of near threshold concentration stimuli. Behav Neurosci. 2012;126:720728. https://doi.org/10.1037/a0030006.

69. Linster C, Nai Q, Ennis M. Nonlinear effects of noradrenergic modulation of olfactory bulb function in adult rodents. J Neurophysiol. 2011; 105:1432-1443. https://doi.org/10.1152/jn.00960.2010.

70. Manella LC, Alperin S, Linster C. Stressors impair odor recognition memory via an olfactory bulb-dependent noradrenergic mechanism. 
Front Integr Neurosci. 2013;7:1-12. https://doi.org/10.3389/fnint. 2013.00097.

71. Gao Y, Strowbridge BW. Long-term plasticity of excitatory inputs to granule cells in the rat olfactory bulb. Nat Neurosci. 2009;12:731-733. https://doi.org/10.1038/nn.2319.

72. Lledo $\mathrm{P}$, Alonso $\mathrm{M}$, Grubb MS. Adult neurogenesis and functional plasticity in neuronal circuits. Nat Rev Neurosci. 2006;7:179-193. https://doi.org/10.1038/nrn1867.

73. Devore S, Linster C. Noradrenergic and cholinergic modulation of olfactory bulb sensory processing. Front Behav Neurosci. 2012;6:1-12. https://doi.org/10.3389/fnbeh.2012.00052.

74. Tong MT, Peace ST, Cleland TA. Properties and mechanisms of olfactory learning and memory. Front Behav Neurosci. 2014;8:1-16. https://doi.org/10.3389/fnbeh.2014.00238.

75. Kovalchuk Y, Hanse E, Kafitz KW, Konnerth A. Postsynaptic induction of bdnf-mediated long-term potentiation. Science. 2002;295:17291734. https://doi.org/10.1126/science.1067766.
76. Park H, Poo M. Neurotrophin regulation of neural circuit development and function. Nat Rev Neurosci. 2013;14:7-23. https://doi.org/ 10.1038/nrn3379.

77. Ramamoorthi K, Fropf R, Belfort GM, et al. Npas4 regulates a transcriptional program in CA3 required for contextual memory formation. Science. 2011;334:1669-1675. https://doi.org/10.1126/science. 1208049.

How to cite this article: Pinho JS, Castilho M, Sollari JS, Oliveira RF. Innate chemical, but not visual, threat cues have been co-opted as unconditioned stimulus for social fear learning in zebrafish. Genes, Brain and Behavior. 2020;e12688. https://doi.org/10.1111/gbb.12688 